\title{
INTRAMURAL VESSELS AND ENDOTHELIAL CELLS IN THE WALLS OF SCLEROSED RETINAL ARTERIES * $\dagger$
}

\author{
BY \\ ARNOLD LOEWENSTEIN \\ From the Tennent Institute, Glasgow (Prof. W. J. B. Riddell).
}

ARterio-SClerotic vessels are clinically and histologically important. I have studied retinal material, especially the tissue of fatty change for a great number of years. The chemical nature of the fat is still practically unknown. I have now studied sclerosed retinal arteries systematically. First I examined the unstained tissue, then I used the technique of retina in bulk. Later I combined the bulk technique with staining, mainly with haematoxylin, Weigert, or sudan red. It seems to be important that the first vasa vasorum shown in the retina of the human eye were in the walls of these sclerosed retinal arteries. (Loewenstein, $194 \pi$ a, b ; 1948 a, b.)

In the literature, intramural vessels in the ascending aorta and in the coronary artery have been described by Robert (1929), who found a discrete capillary system in the intima of the aorta. Paterson (1936) mentioned vascularization of coronary arteries in association with arterio-sclerosis. It is most important that Paterson never discovered intramural vessels in normal arteries. As far as I understand him, he did not find any newly formed vessels in the arteries either of humans or of normal animals.

Winternitz and others (1938), produced a perfect specimen demonstrating the vasa vasorum in sclerosed coronary arteries. The tissue of the arteries was cleared and injected with black ink, and showed the presence of a typical network of vasa vasorum in the rich vascular system of the intima. Of course, it is much easier to work on these huge arteries than on our small retinal vessels. But I frequently found intramural vessels in the retina, especially as our technique improved. The fine sclerosed vessels came out very clearly, especially with reduced light and frequent change of the iris diaphragm.

We know, of course, that cases of arterio-sclerosis appear clinically as a ramified rete mirabile. Ballantyne (1949) has shown typical retinal and preretinal vessels by ophthalmoscopic examination. In our histological material we demonstrated new vessels, the "vasa vasorum". This material was mostly arterio-

* Keceived for publication April 3, 1950.

+ This research was sponsored by the W. H. Ross Foundation (Scotland) for the Study of the Prevention of Blindness. 
sclerotic with hypertension, but vasa vasorum could be shown quite frequently in diabetes, and also in thrombosis of the central vein, often combined with arterio-sclerosis. We discovered them in three cases of periarteritis nodosa and in two cases of vasculitis of young adults with haemorrhages in the retina and vitreous. These intramural vessels are most interesting but not fully understood either in periarteritis nodosa or in vasculitis.

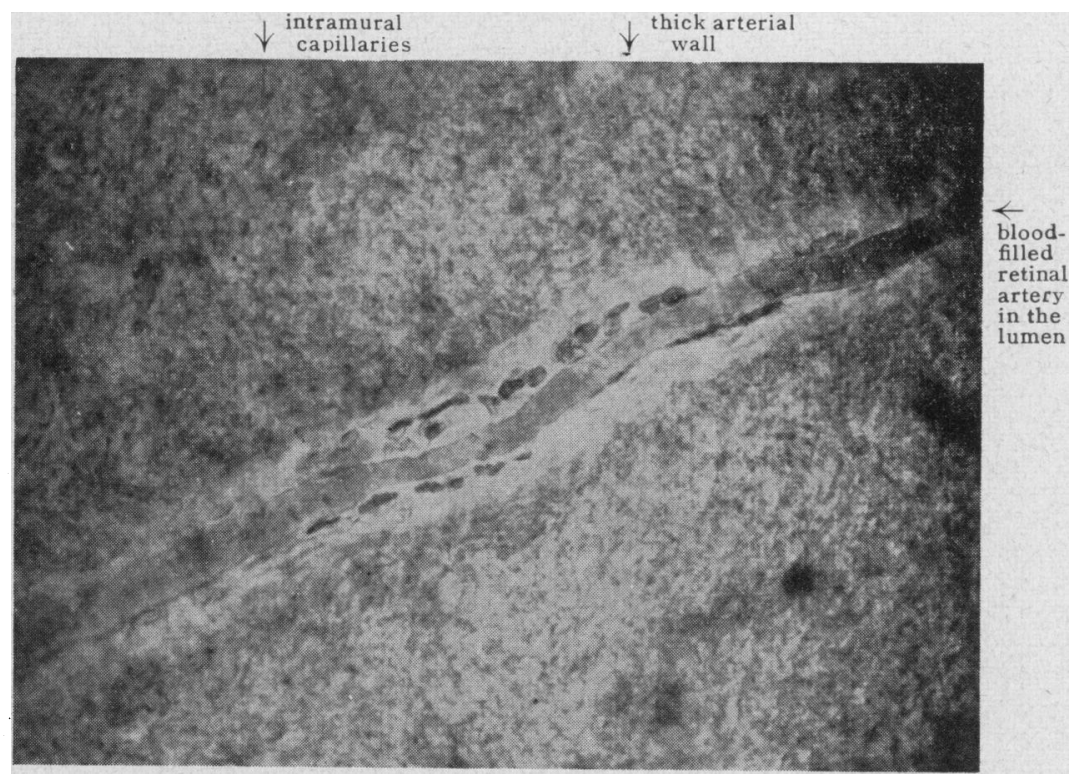

FIG. 1.-Intramural vessels. Betina in bulk, with thrombosis of the central vein. The artery in the retina shows the thickened wall. Haematoxylin. $300 \times$.

The retinal tissue must be carefully cleaned with a brush to remove the blood, then stained as described, and kept in glycerine. Later the material is examined in glycerine jelly. When we study the retinal tissue in bulk we look for the lumen of the artery and its wall (Fig. 1). In bulk the ganglion cells of the retina appear dark blue. We recognize the lumen which is mostly filled with red blood corpuscles, variable in diameter, sometimes dilated, sometimes narrow. These sclerosed walls appear whitish, swollen, and irregular. The intramural capillaries are sharply delineated and filled with thin red blood corpuscles (Fig. 1); they are perfectly visible in bulk. The oblong blood corpuscles are characteristic for the thin intramural vessels where they frequently run along the lumen. Other intramural capillaries are arranged in spirals or transversely (Fig. 6). These intramural capillaries are most 
frequently present in the area of the whitish sclerosed walls on both sides of the red lumen with its round red blood corpuscles (Fig. 1).

These fine capillaries in the wall were stained in bulk in a tissue of exactly $0.5 \mathrm{~mm}$. in length and photographed. There was no intramural vessel on either side of this part of the artery.

That part of the artery with the intramural vessels was then embedded in paraffin and cut in serial sections. We studied especially sections 230 to 280 where we found the intramural vessels exactly in the wall. In these sections one sees the capillaries filled with red blood corpuscles (Fig. 2); there are no capillaries before 230 or after 280 . The vasa vasorum only appear in the piece of artery between these two sections (Fig. 2 a).

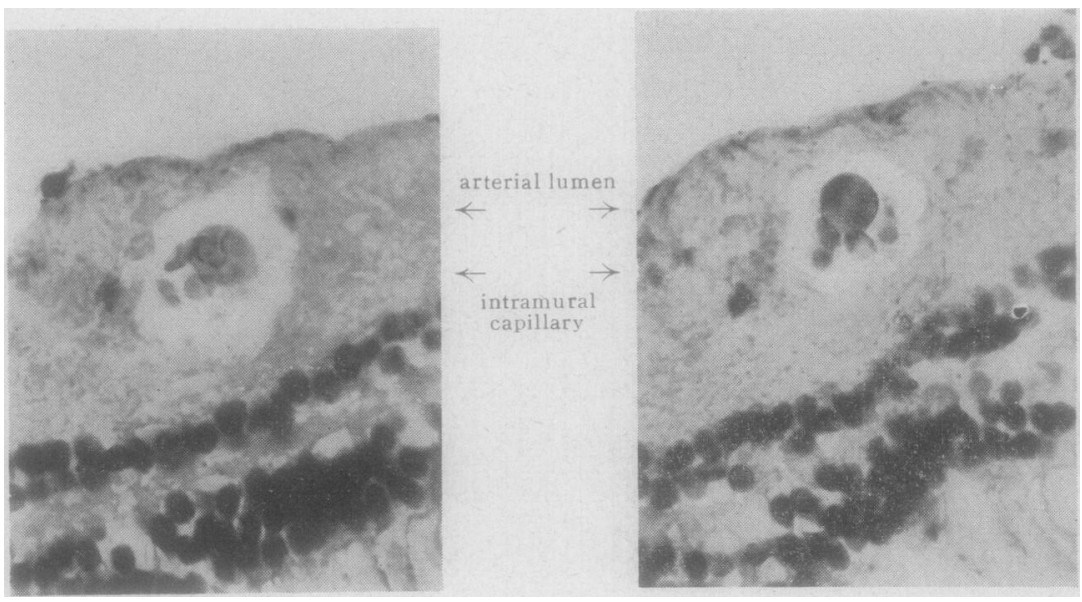

FIG. $2(a)$ and $(b)$. - Intramural vessels in paraffin sections, showing sclerosed walls. Intramural capillaries filled with red blood corpuscles. Series between 230 and 280 , single 50 sections of $10 \mu=0.5 \mathrm{~mm}$.

In the sections before 230 and after 280 , where there were no intramural capillaries, we found endothelial cells which are certainly pathological (Fig. $3 a$ and $b$ ). I cannot offer an explanation for these endothelial cells; they might be growing from the endothelium of the lumen or be newly growing capillaries without any red blood corpuscles. We have already seen endothelial cells growing out of the lumen of arteries in previous sections. It is possible that the blood fills the intramural vessels at a later stage (Fig. $3 c$ and $d$ ). In any case these endothelial cells in the pathological walls require intensive study in bulk as well as in sections.

In one of the cases of periarteritis nodosa there can be seen part of the thrombosed vessel overbridged by newly formed intramural vessels (Fig. 4). 
Fig. 5 shows a sclerosed vessel with intramural capillaries and fatty changes. The tissue was stained in bulk with haematoxylin and sudan red, and the fat in the walls appears shining red. It is quite frequent to find both processes in the sclerosed vesselsintramural vessels and fatty change.

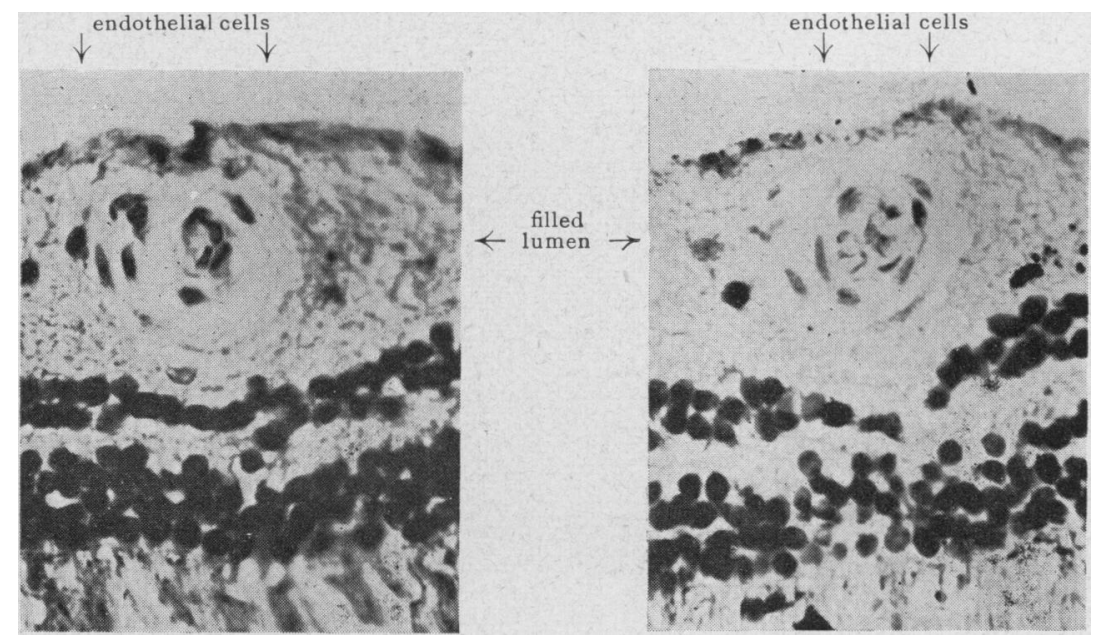

FIG. $3(a)$ and (b).- Sclerosed retinal artery. Lumen filled with red blood corpuscles. Endothelial cells in the sclerosed wall.

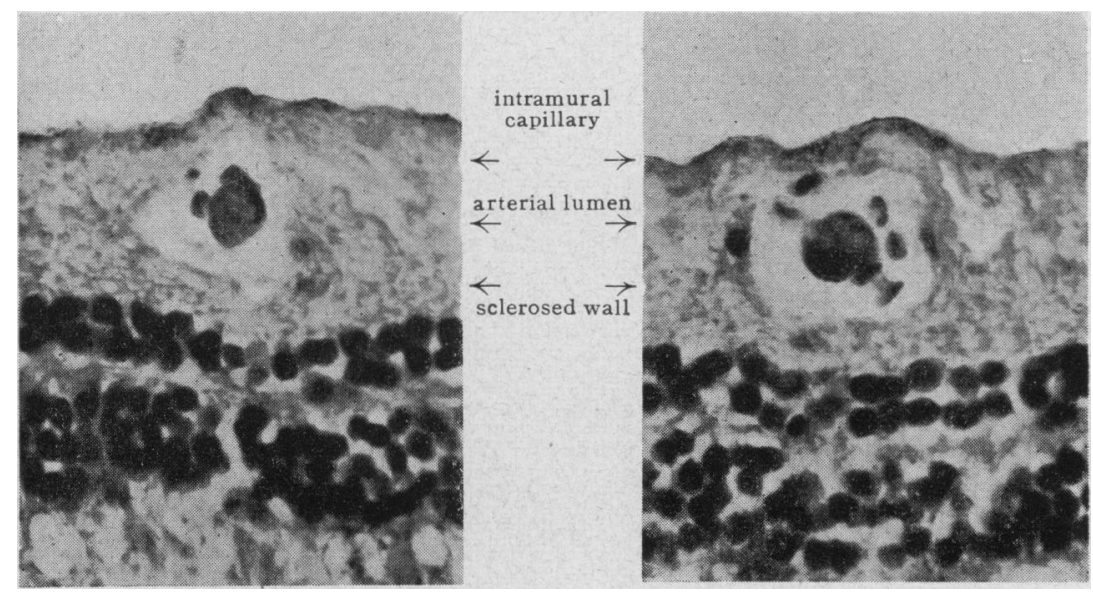

FIG. $3(c)$ and (d).--Intramural capillaries with red blood corpuscles.

So far I have described intramural vessels in arteries rather than in veins; but we found vasa vasorum in the walls of two crossing vessels. I therefore conclude that intramural vessels 


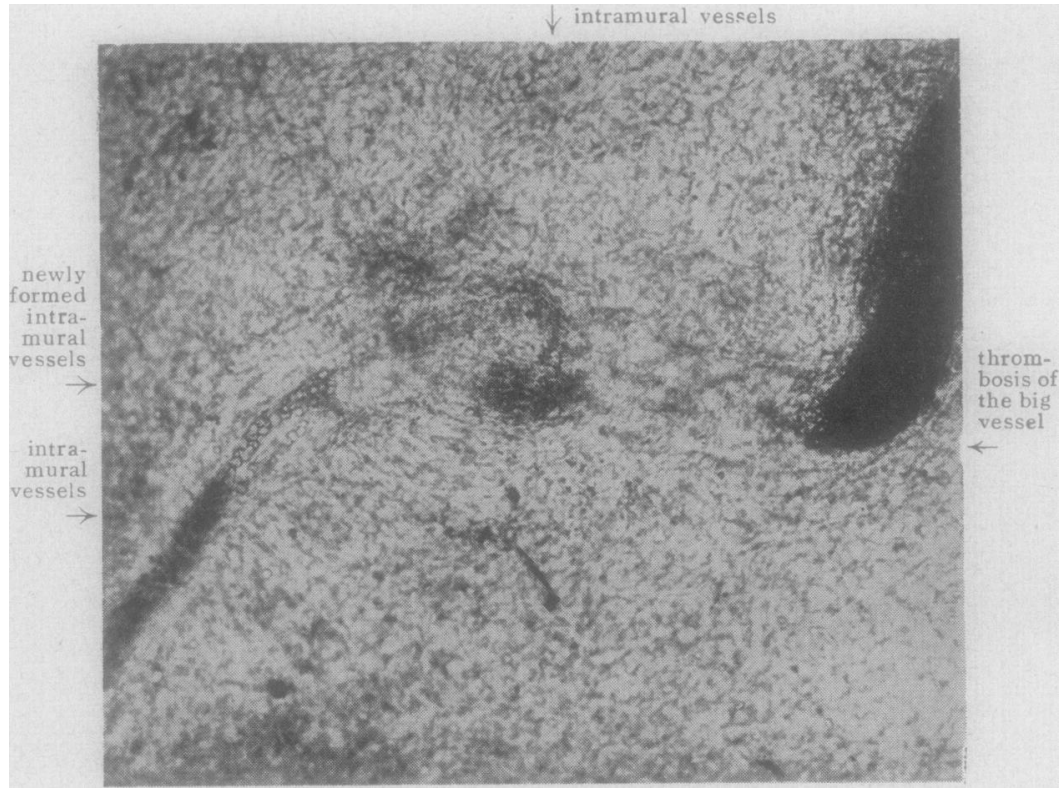

FIG. 4.-Periarteritis nodosa. Retina in bulk. The thrombotic process has produced a new formation of overbridging vessels. Anilin blue. $150 \times$ approx.

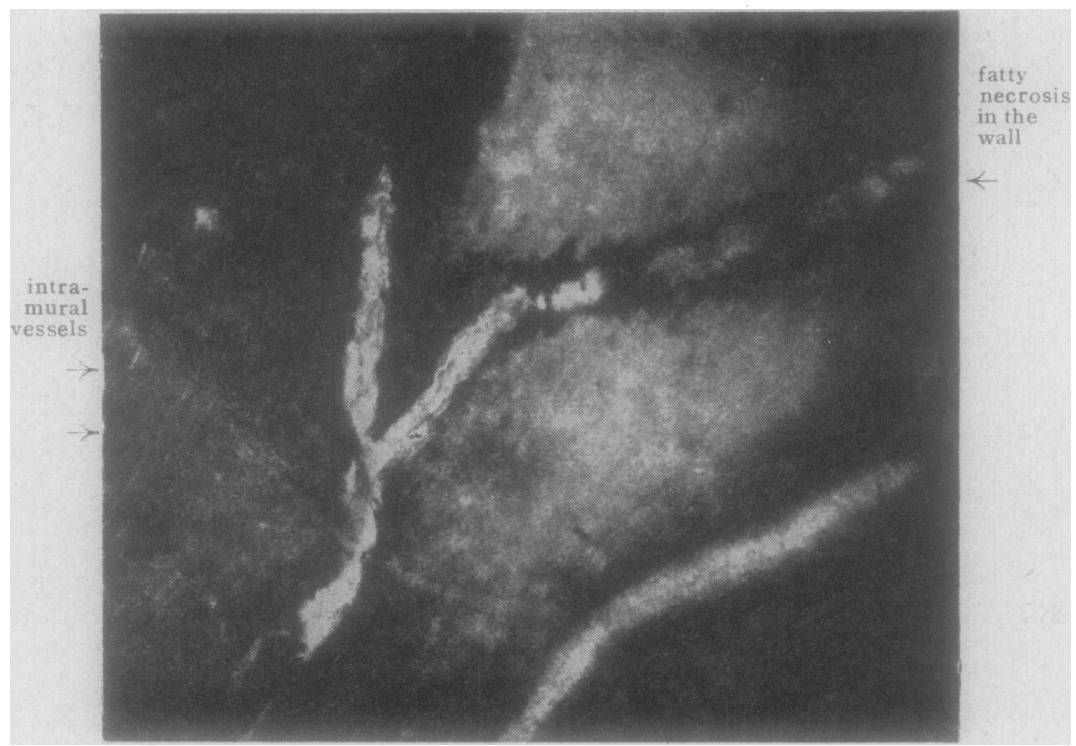

FIG. 5.-Hypertensive and diabetic arteries containing intramural vessels. Whole wall filled with fatty necrosis. Stained in bulk, haematoxylin and scarlet red. $150 \times$. 
are also present in veins. The vessels in veins and in arteries have a different appearance. The thrombotic closure of veins leads to the formation of by-passing vessels within the walls of the vein. In the sclerosed artery, however, those intramural vessels do not by-pass any particular part of the lumen, but ramify in the walls of the parent vessel. Figs. $6(a)$ and $(b)$ especially show the clear difference in the two modes of new-vessel formation; Fig. $6(a)$ shows the new vessels spiralling round the parent vessel and ramifying in the substance. of the walls, while Fig. $6(b)$ shows the more direct course of the vasa vasorum in the occluded vein.

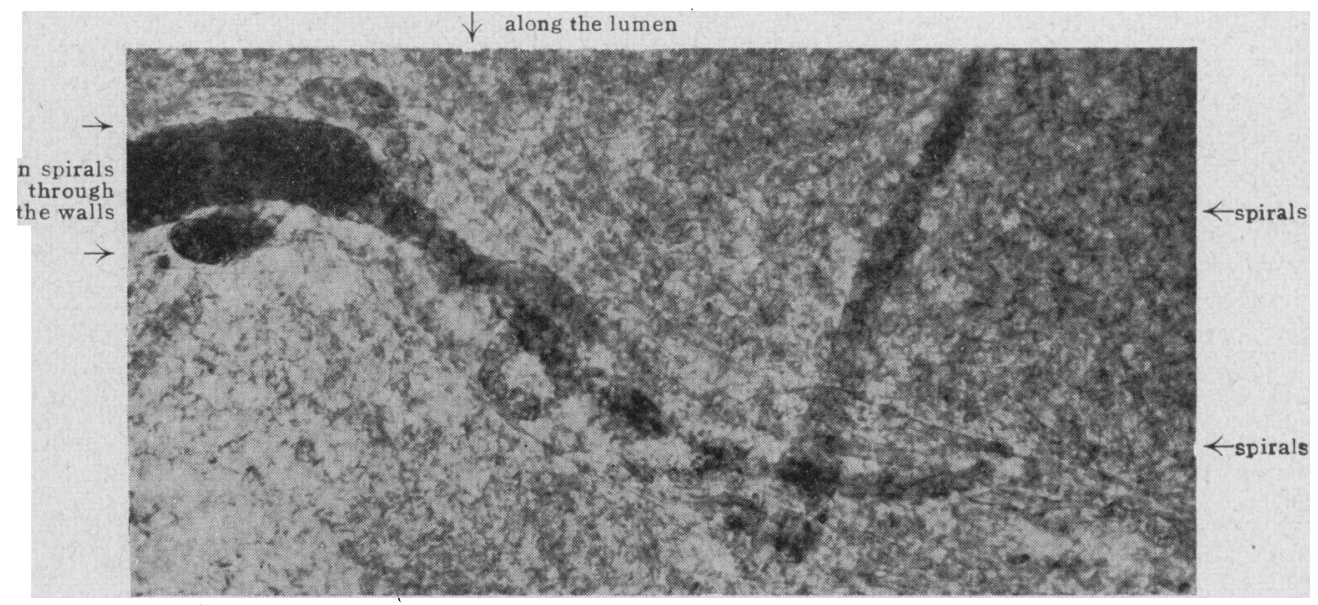

FIG. $6(a)$-Intramural vessels (arteries)-in the retina-in spirals through the wall.

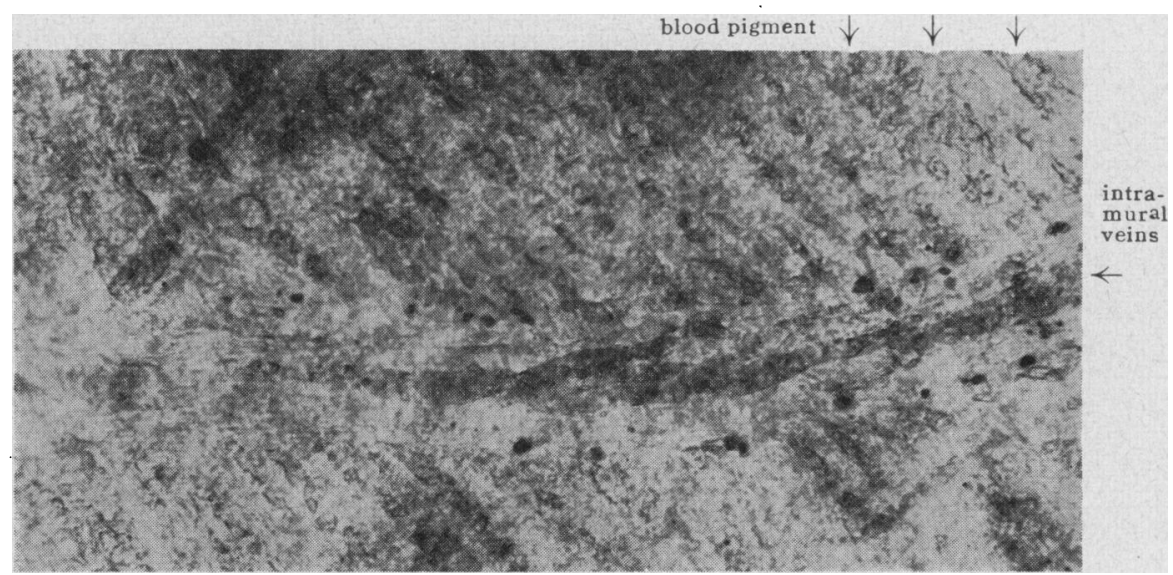

FIG. $6(b)$.- Intramural vessels (veins) in thrombosis. 
Hitherto I have discussed intramural vessels in the aorta and coronary arteries, and in the arteries of the retina. There is a probability that the vessels of other tissues have different vasa vasorum. I have tried to find these in brain tissue, using different techniques, such as injecting the medium-sized vessels with ink, or clearing the very fine cerebral vessels, but so far without success.

I have previously shown the vasa vasorum in the retina in sketches. The microscopical stereoscopical depth appears in bulk vessels between 30 and $40 \mu$ (Loewenstein, 1948). This communication contains photomicrographs only; they are in different positions, some high, some deep.

\section{Conclusion}

(1) Intramural vessels are shown in photomicrographs of the retina in bulk. One shows an intramural vessel $0.5 \mathrm{~mm}$. in length.

(2) Some of these intramural arteries are shown in serial paraffin sections of the same length $(0.5 \mathrm{~mm}$.).

(3) Endothelial cells are visible in the sclerosed arterial wall. These endothelial cells might possibly be capillaries without red blood cells.

(4) Intramural vessels are present in both arteries and veins.

(5) Intramural vessels are shown in a case of periarteritis nodosa.

I am indebted to Mr. John Watt for his help in histological work and for his photo micrographs.

\section{REFERENCES}

Ballantyne, A. J. (1949). Ophthalmologica, Bascl, 118, 611. Loewenstein, A. (1947a). Nature, Lond., 160, 124.

- (1947b). Trans.ophthal. Soc. U.K., 66, 581.

(1948a). Arch. Ophthal., Chicago, 39, 9.

(1948b). British Journal of Ophthalmology, 32, 748.

Paterson, J, C. (1936). Arch. Path., 22, 313.

Robertson, H. F. (1929). Ibid., 8, 881.

Winternit $Z$, M. C.. Thomas, R. M., and LeCompte, P. M. (1938). "The Biology of Arteriosclerosis". Thomas, Springfield, Ill. 